\title{
Validation of 1-hour post-thyroidectomy parathyroid hormone level in predicting hypocalcemia
}

\author{
Trung N Le $1^{1 *}$, Paul D Kerr ${ }^{2}$, Donna E Sutherland ${ }^{2}$ and Pascal Lambert ${ }^{3}$
}

\begin{abstract}
Background: Prior work by our group suggested that a single one hour post-thyroidectomy parathyroid hormone (1 hr PTH) level could accurately stratify patients into high and low risk groups for the development of hypocalcemia. This study looks to validate the safety and efficacy of a protocol based on a $1 \mathrm{hr}$ PTH threshold of $12 \mathrm{pg} / \mathrm{ml}$.

Study design: Retrospective analysis of consecutive cohort treated with standardized protocol.

Methods: One hundred and twenty five consecutive patients underwent total or completion thyroidectomy and their PTH level was drawn 1-hour post operatively. Based on our previous work, patients were stratified into either a low risk group (PTH $<12 \mathrm{pg} / \mathrm{ml}$ ) or a high risk group (PTH $\geq 12 \mathrm{pg} / \mathrm{ml})$. Patients in the high risk group were immediately started on prophylactic calcium carbonate $(5-10 \mathrm{~g} / \mathrm{d})$ and calcitriol $(0.5-1.0 \mathrm{mcg} / \mathrm{d})$. The outcomes were then reviewed focusing mainly on how many low risk patients developed hypocalcemia (false negative rate), and how many high risk patients failed prophylactic therapy.

Results: Thirty one patients (25\%) were stratified as high risk, and 94 (75\%) as low risk. Five (16\%) of the high risk patients became hypocalcemic despite prophylactic therapy. Two of the low risk group became hypocalcemic, (negative predictive value $=98 \%$ ). None of the hypocalcemic patients had anything more than mild symptoms.

Conclusions: A single 1-hour post-thyroidectomy PTH level is a very useful way to stratify thyroidectomy patients into high and low risk groups for development of hypocalcemia. Early implementation of oral prophylactic calcium and vitamin $D$ in the high risk patients is a very effective way to prevent serious hypocalcemia. Complex protocols requiring multiple calcium and PTH measurements are not required to guide post-thyroidectomy management.
\end{abstract}

Keywords: Parathyroid hormone, Hypocalcemia, Total thyroidectomy, Completion thyroidectomy, Calcium, Calcitriol, 1-hour PTH

\section{Background}

Hypocalcemia is one of the most common complications following total thyroidectomy, occurring in $10-50 \%$ of cases [1]. It is usually transient, but can be permanent in $0.5-10.6 \%$ of patients. It is caused by parathyroid devascularization, stunning, or incidental removal of the parathyroid gland(s) [1-6].

The nadir for hypocalcemia typically occurs at around $24-48 \mathrm{~h}$ postoperatively but may be as delayed as post-op

\footnotetext{
* Correspondence: trungngocle@gmail.com

'Otolaryngology Head \& Neck Surgery, University of Manitoba, Health Sciences Centre, GB421-820 Sherbrook Street, Winnipeg, MB R3A 1R9, Canada

Full list of author information is available at the end of the article
}

day 4 [7]. Therefore, detecting patients requiring calcium replacement therapy with serial calcium measurements can take multiple blood tests over several days. Placing all patients on calcium therapy unnecessarily commits many patients to unnecessary treatment and puts them at risk for hypercalcemia [8]. A clinical laboratory method for early prediction of postoperative hypocalcemia could, therefore, facilitate earlier implementation of treatment, and early discharge ( $\leq 24$ hours).

In recent years, multiple retrospective and prospective studies have emerged, which support the use of postoperative serum parathyroid hormone $(\mathrm{PTH})$ levels as accurate predictors of hypocalcemia in postoperative thyroidectomy patients. Different groups have published

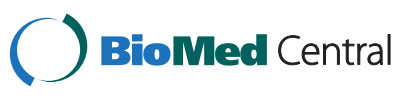

(c) 2014 Le et al.; licensee BioMed Central Ltd. This is an open access article distributed under the terms of the Creative Commons Attribution License (http://creativecommons.org/licenses/by/2.0), which permits unrestricted use, distribution, and reproduction in any medium, provided the original work is properly cited. 
considerable research on this topic, demonstrating that 1 and 6-hour postoperative PTH and Calcium levels had a high sensitivity and specificity of detecting postoperative hypocalcemia $[9,10]$. Other studies compared PTH percent change as predictor for hypocalcemia [11-13].

Our group previously published a study in which we prospectively followed 42 patients postoperatively with serial PTH and $\mathrm{Ca}$ measurements [17]. We found that a that a single postoperative parathyroid hormone (PTH) level, drawn 1 hour after total thyroidectomy, accurately correlated with the development of significant hypocalcemia. All patients who exhibited a PTH level of less than $9 \mathrm{pg} / \mathrm{mL} 1$ hour after surgery developed symptomatic and biochemical hypocalcemia requiring treatment with calcium and vitamin D. None of the patients with $\mathrm{PTH} \geq 9$ developed hypocalcemia.

Subsequent unpublished data from 100 consecutive total and completion thyroidectomy patients revealed that approximately half of the patients with 1-hour postthyroidectomy PTH (1 hr PTH) levels in the range of 9-12 pg/ml will develop hypocalcemia. Our center currently categorizes post-thyroidectomy patients as high risk for developing hypocalcemia based on a $1 \mathrm{hr}$ PTH of less than $12 \mathrm{pg} / \mathrm{ml}$. The high risk group is started on prophylactic calcium and vitamin D therapy as soon as the $1 \mathrm{hr}$ PTH level is determined. Those with $1 \mathrm{hr}$ PTH levels of $12 \mathrm{pg} / \mathrm{ml}$ or higher are deemed low risk, and are not given prophylactic treatment.

The purpose of this study is to determine the number of high-risk patients who develop hypocalcemia after prophylactic treatment, and to determine the number of low-risk patients who develop hypocalcemia without prophylactic treatment.

\section{Methods}

The study was a retrospective, cohort chart review of consecutive patients undergoing completion or total thyroidectomy from July 2008 to July 2012. All patients underwent a PTH measurement 1 hour post surgery. Based on the result of the $1 \mathrm{hr} \mathrm{PTH}$, patients were divided into a high risk group, $(\mathrm{PTH}<12 \mathrm{pg} / \mathrm{ml}-$ at high risk for developing hypocalcemia), and a low risk group ( $\mathrm{PTH} \geq$ $12 \mathrm{pg} / \mathrm{ml}$ - at low risk for developing hypocalcemia).

Patients in the high risk group were started on prophylactic supplementation including calcium carbonate (total dose 5-10 grams per day) and calcitriol (total dose 0.5-1.0 mcg per day) as soon as the PTH level was determined (i.e. within a few hours of surgery). Patients in the low risk group were not given the supplementation.

Both groups were candidates for same day or 23 hour discharge, unless symptoms developed in the interim. All patients were seen in follow-up 1-4 weeks postoperatively. Calcium and PTH levels were rechecked at that time in high risk group to determine the need for ongoing calcium and vitamin D supplementation.

Patient information regarding age, sex, procedure type, final thyroid pathology, and presence of symptoms of hypocalcemia were recorded.

The biologically active, intact 84 amino acid PTH level was measured using the Immulite assay (Diagnostic Products Corporation, Los Angeles, CA), a sensitive and specific two site immunochemiluminometric test. The total incubation time for the Immulite assay is 1520 minutes, with an analytical sensitivity of 2 to $3 \mathrm{pg} / \mathrm{mL}$. The normal PTH level in our laboratory is 7 to $50 \mathrm{pg} / \mathrm{mL}$. Test results are available shortly after 1 hour from initial sampling. The study was approved by the hospital research ethics committee (Institution Review Board: University of Manitoba Bannatyne Campus Research Ethics Board).

\section{Surgical technique}

Surgical techniques for total and completion thyroidectomy were similar for each operation and all procedures were performed by one of three fellowship-trained head and neck surgeons. Parathyroid glands were preserved whenever possible.

\section{Overall outcomes}

Patients ultimately fell into 4 groups:

1. High risk, eucalcemic (adequate replacement) These patients had $1 \mathrm{hr}$ PTH level $<12 \mathrm{pg} / \mathrm{ml}$, received calcium and calcitriol supplementation, did not develop symptoms of hypocalcemia, did not have documented biochemical hypocalcemia (corrected calcium $\leq 1.9 \mathrm{mmol} / \mathrm{l}$ ), and did not require increased supplementation.

2. High risk, hypocalcemic (inadequate replacement) These patients had $1 \mathrm{hr}$ PTH level $<12 \mathrm{pg} / \mathrm{ml}$, received calcium and calcitriol supplementation, but developed symptoms of hypocalcemia, had documented biochemical hypocalcemia (corrected calcium $\leq 1.9 \mathrm{mmol} / \mathrm{l}$ ), and required increased supplementation.

3. Low risk, eucalcemic (true negative prediction of hypocalcemia) These patients had $1 \mathrm{hr}$ PTH level $\geq 12 \mathrm{pg} / \mathrm{ml}$, and did not develop symptoms of hypocalcemia, did not have documented biochemical hypocalcemia, and did not require supplementation.

4. Low risk, hypocalcemic (false negative prediction of hypocalcemia)

These patients had $1 \mathrm{hr}$ PTH level $\geq 12 \mathrm{pg} / \mathrm{ml}$, but developed symptoms of hypocalcemia, had documented biochemical hypocalcemia, and required calcium replacement. 


\section{Primary endpoints}

We defined two primary endpoints for our study:

1. How many patients do we place at risk by falsely categorizing them as low risk: Negative predictive value of a $1 \mathrm{hr}$ PTH level of $\geq 12 \mathrm{pg} / \mathrm{ml}$ ?

2. How often do prophylactic oral calcium and vitamin D supplements fail in the high risk group?

\section{Results}

In reviewing of our July 2008 - July 2012 data, there were 125 patients who underwent completion or total thyroidectomy (Figure 1); 94 females and 31 males. One hundred and nine patients underwent total thyroidectomy and 16 underwent completion thyroidectomy. The mean age was 54 years old with a range of $20-87$ years. Most procedures were done for oncologic indications. Final histologic diagnoses included 24 multinodular goiters, 3 Grave's disease, 3 follicular adenomas, and 95 malignancies.

Of the 125 patients, 31 (25\%) had a 1 hr PTH level < $12 \mathrm{pg} / \mathrm{ml}$, and were therefore deemed to be at high risk for developing hypocalcemia. Despite this group receiving supplemental calcium and calcitriol, 16\% (5/31) developed hypocalcemia (Figure 1). None of these patients developed serious sequelae from hypocalcemia such as tetany or dysrhythmia.

Niney-four patients (75\%) had a 1 hr PTH level $\geq$ $12 \mathrm{pg} / \mathrm{ml}$, and were thus classified as low risk for developing hypocalcemia. Two of these patients (2\%) developed hypocalcemia. Thus, the negative predictive value of a $1 \mathrm{hr} \mathrm{PTH} \geq 12 \mathrm{pg} / \mathrm{ml}$ is $98 \%$ (Figure 1). Again, the hypocalcemic patients had only mild symptoms. None of the patients that became hypocalcemic had serious sequelae.

\section{Discussion}

Many authors [9-16] have demonstrated the correlation between post-thyroidectomy PTH levels and the development of hypocalcemia. However, the ensuing management protocols have generally been cumbersome; requiring repeated blood tests and the use of graphs or formulas. Previously published work by our group [17] indicated that a postoperative $\mathrm{PTH}$ level $<9 \mathrm{pg} / \mathrm{ml}$, drawn 1 hour after total thyroidectomy, accurately correlated with the development of significant hypocalcemia, leading us to believe that a simpler protocol could be developed. Our unpublished data of 100 subsequent patients between 2003-2007 revealed that approximately half the patients with postoperative PTH levels between 9-12 $\mathrm{pg} / \mathrm{ml}$ will develop hypocalcemia. Therefore, we have raised the PTH cut-off level from 9 to $12 \mathrm{pg} / \mathrm{ml}$ in the development of a protocol for the of a single 1-hour PTH level in predicting the risk of development of hypocalcemia.

Our protocol has an excellent negative predictive value of $98 \%$. It is important to note that the two low risk patients that developed hypocalcemia had borderline PTH levels of 12 and $13 \mathrm{pg} / \mathrm{ml}$. The resulting hypocalcemia did not result in any serious sequelae. One of these two patient became symptomatic within one week of surgery and was started on supplementation. Interestingly, in the second postoperative week they became hypercalemic and the supplementation was stopped. The other developed post-operative hypocalcemia in association with significant hypomagnesemia. This patient also required prolonged hospital stay and intravenous calcium and magnesium supplementation. This case illustrates the potential for other factors such as malnutrition and hypomagnesemia to potentially exacerbate calcium imbalance in these patients. Perhaps magnesium levels should be incorporated into the perioperative management regimen.

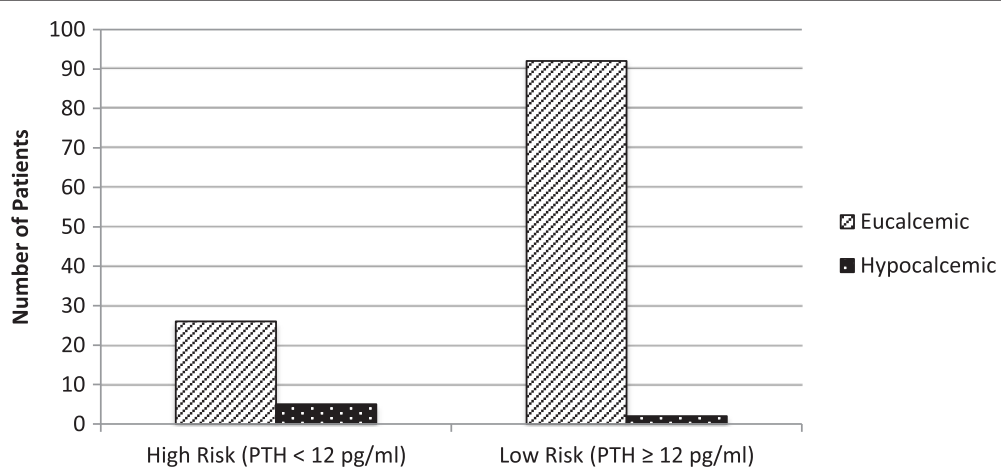

Figure 1 Proportion of patients in high and low risk groups developing hypocalcemia:Patients who developed hypocalcemia in the high risk group (5/31) were identified as being likely to develop hypocalcemia, but failed to adequately respond to the initial oral prophylactic calcium and vitamin D supplementation. Patients who developed hypocalcemia in the low risk group are those who were inaccurately categorized by our PTH cut-off (2/94). All the patients who developed hypocalcemia had only mild symptoms. There were no serious sequelae in any patients. 
While the PTH cut-off could be raised to accommodate these patients, this will inevitably result in a greater number of patients being unnecessarily started on prophylactic treatment. We prefer to leave these borderline patients untreated, but warn them to be extra vigilant about reporting any symptoms so that calcium testing can be promptly performed.

The positive predictive value of our protocol cannot be formally validated with this study as all the high risk patients were given prophylactic treatment. Therefore, it is impossible to determine how many high risk patients would have gone on to develop hypocalcemia. However, we know from our previous series and from literature [1-6] in which high risk patients were not treated that approximately $25 \%$ of patients will develop symptoms. This correlates very well with the proportion of patients that our PTH cut-off is identifying as high risk. Therefore, we feel that the positive predictive value is very high, likely in excess of $90 \%$. A high positive predictive value means that very few patients are being unnecessarily placed on supplemental medication or requiring unnecessary follow-up bloodwork.

Our protocol of immediately implementing prophylactic calcium and activated vitamin D supplementation in the high risk group appears to be very effective. From our previous studies, we know that the vast majority of the patients in the high risk group develop symptomatic hypocalcemia if not treated with supplemental calcium and vitamin D. By combining the data of our current study with information from our previous study [17] in which patients from the high risk group were purposefully observed without prophylactic treatment, we found a significant decrease in hypocalcemia among high risk patients receiving prophylactic treatment $(\mathrm{OR}=0.05$; 95\% CI: 0.01- 0.12; p <.001)

While our protocol of prophylactic supplementation failed to prevent hypocalcemia in $16 \%$ of high risk patients, it is important to note that none of the patients suffered anything more than mild symptoms. There were no serious sequelae.

We have analyzed the 5 cases that were deemed to have failed prophylactic therapy. Factors that appear to be involved in failed supplementation include: compliance, lower doses of calcium and vitamin D, and dietary deficiencies such as hypomagnesemia. All of these patients developed hypocalcemic symptoms within 10 days of surgery. $60 \%(3 / 5)$ of these five patients developed postoperative hypocalcemia associated with documented hypomagnesemia. These patients required prolonged hospital admission or readmission for intravenous calcium and magnesium supplementations. This finding emphasized the importance of magnesium in calcium metabolism. 20\% (1/5) of these five patients continue to have long-term hypoparathyroidism (>6 months) and required ongoing calcium and calcitriol supplementations. Some of these patients were strict vegetarians, had low magnesium and/or low vitamin D levels pre-op. This association has been documented in the literature [18].

There was a range in the initial prohylactic supplementation of $5-10 \mathrm{~g}$ calcium carbonate and $0.5-1 \mathrm{mcg}$ calcitriol. The five high risk patients that became hypocalcemic were started on the lower end of oral supplementations (5.0 g of calcium and $0.5 \mathrm{mcg}$ of calcitriol per day). Perhaps, we should be routinely dosing between 7.5-10 g/d of calcium carbonate and $0.75-1 \mathrm{mcg} / \mathrm{d}$ of calcitriol .

One of the high risk patients developed hypercalcemia on oral supplementation. Therefore, we recommend that a calcium level be drawn 1-2 weeks postop in the high risk group so as to assist in judging the rate at which medication can be tapered. This is echoed by other authors [19].

No predictor of hypoparathyroidism is perfectly accurate. Whatever method of risk categorization is chosen, it is best to consider the result in the full clinical context. How confident is the surgeon regarding the preservation of viable parathyroid glands? What is the preoperative nutritional and biochemical state of the patient? What is the anticipated compliance of the patient? All of these factors can potentially be taken into consideration so as to further lessen risk and improve patient outcomes.

It is important to educate patients regarding to signs and symptoms of hypocalcemia. Our protocol eliminates the need for multiple unnecessary blood tests, or the need for complex formulas and protocols postoperatively. A single 1-hour post-thyroidectomy PTH level of $<12 \mathrm{pg} / \mathrm{ml}$ is a very good predictor of hypocalcemia. Complex protocols requiring multiple calcium and PTH measurements are not required to guide post-thyroidectomy management. This simple protocol provides the risk stratification required to safely employ selective calcium and calcitriol supplementation. This facilitates safe, early discharge with informative postoperative instructions for patient and firm follow-up plan.

\section{Conclusions}

A single 1-hour post-thyroidectomy PTH level is a valuable tool for stratifying patients as to their level of risk for developing hypocalcemia. Patients that are deemed high risk should be immediately started on prophylactic supplementation, and can safely be considered for 23hour discharge. Those deemed low risk can be safely discharged without any supplementation.

\section{Abbreviations}

PTH: Parathyroid hormone; LOS: Length of hospital stay; pg: Picogram; ml: Milliliter; mcg: Microgram; g: Gram; mmol: Millimole; L: Liter;

ER: Emergency department; hr: Hour; post-op: Postoperative; cCa: Corrected cacium (based on albumin level); D/C: Discharge home.

\section{Competing interests}

The authors declare that they have no competing interests. 


\section{Authors' contributions}

TL and PK collected and analysed data, and drafted the manuscript.

$T L$ and DS applied for research ethics board. PL provided statistical analysis.

All authors read and approved the final manuscript.

\section{Acknowledgements}

We would like to thank Dr. Raza Naweed and Miss Emi Okamoto for data acquisition.

\section{Author details}

${ }^{1}$ Otolaryngology Head \& Neck Surgery, University of Manitoba, Health Sciences Centre, GB421-820 Sherbrook Street, Winnipeg, MB R3A 1R9, Canada. ${ }^{2}$ Head and Neck Oncology, University of Manitoba, Health Sciences Centre, GB421-820 Sherbrook Street, Winnipeg, MB R3A 1R9, Canada. ${ }^{3}$ Health Outcomes Analyst, Cancer Care Manitoba, ON2114-675 McDermot Avenue, Winnipeg, MB R3E 0 V9, Canada.

Received: 3 April 2013 Accepted: 26 January 2014

Published: 29 January 2014

\section{References}

1. Pattou F, Combemale F, Fabre S, Carnaille B, Decoulx M, Wemeau JL, Racadot A, Proye C: Hypocalcemia following thyroid surgery: incidence and prediction of outcome. World J Surg 1998, 22:718-724

2. Asari R, Passler C, Kaczirek K, Scheuba C, Niederle B: Hypoparathyroidism after total thyroidectomy: a prospective study. Arch Surg 2008, 143:132-138.

3. McHenry CR, Speroff T, Wentworth D, Murphy T: Risk factors for postthyroidectomy hypocalcemia. Surgery 1994, 116:641-648.

4. Erbil Y, Bozbora A, Ozbey N, Issever H, Aral F, Ozarmagan S, Tezelman S: Predictive value of age and serum parathormone and vitamin $d 3$ levels for postoperative hypocalcemia after total thyroidectomy for nontoxic multinodular goiter. Arch Surg 2007, 142:1182-1187.

5. Sywak MS, Palazzo FF, Yeh M, Wilkinson M, Snook K, Sidhu SB, Delbridge LW: Parathyroid hormone assay predicts hypocalcaemia after total thyroidectomy. ANZ J Surg 2007, 77:667-670.

6. Walker Harris V, Jan De Beur S: Postoperative hypoparathyroidism: medical and surgical therapeutic options. Thyroid 2009, 19:967-793.

7. Lo Gerfo P, Gates R, Gazetas P: Outpatient and short-stay thyroid surgery. Head Neck 1991, 13:97-101.

8. Roh $\mathrm{J}$, Park Cl: Routine oral calcium and vitamin D supplements for prevention of hypocalcemia after total thyroidectomy. Am J Surg 2006, 192:675-678.

9. Payne RJ, Tewfik MA, Hier MP, Tamilia M, Mac Namara E, Young J, Black MJ: Benefits resulting from 1 and 6-hour parathyroid hormone and calcium levels after thyroidectomy. Otolaryngol Head Neck Surg 2005, 133:386-390.

10. Payne RJ, Hier MP, Tamilia M, Mac Namara E, Young J, Black MJ: Same-day discharge after total thyroidectomy: the value of 6-hour serum parathyroid hormone and calcium levels. Head Neck 2005, 27:1-7.

11. Chapman DB, French CC, Leng X, Browne JD, Waltonen JD, Sullivan CA: Parathyroid hormone early percent change: an individualized approach to predict postthyroidectomy hypocalcemia. Am J Otolaryn H\&N Med \& Surg 2012, 33:216-220

12. Vescan A, Witterick I, Freeman J: Parathyroid hormone as a predictor of hypocalcemia after thyroidectomy. Laryngoscope 2005, 115:2105-2108.

13. Scurry WC, Beus KS, Hollenbeak CS, Stack BC Jr: Perioperative parathyroid hormone assay for diagnosis and management of postthyroidectomy hypocalcemia. Laryngoscope 2005, 115:1362-1366.

14. Noordzij JP, Lee SL, Bernet VJ, Payne RJ, Cohen SM, McLeod IK, Hier MP, Black MJ, Kerr PD, Richards ML, Lo CY, Raffaelli M, Bellantone R, Lombardi $C P$, Cohen Jl, Dietrich MS: Early prediction of hypocalcemia after thyroidectomy using parathyroid hormone: an analysis of pooled individual patient data from nine observational studies. J Am Coll Surg 2007, 205:748-754

15. Cayo AK, Yen TW, Misustin SM, Wall K, Wilson SD, Evans DB, Wang TS: Predicting the need for calcium and calcitriol supplementation after total thyroidectomy: results of a prospective, randomized study. Surgery 2012, 152:1059-1067.

16. Huang SM: Do we overtreat post-thyroidectomy hypocalcemia? World J Surg 2012, 36:1503-1508.
17. Lam A, Kerr PD: Parathyroid hormone: an early predictor of postthyroidectomy hypocalcemia. Laryngoscope 2003, 113:2196-2200.

18. Nhan C, Dolev Y, Mijovic T, Rivera JA, Kallai-Sanfaçon MA, Mlynarek AM, Payne RJ: Vitamin D deficiency and the risk of hypocalcemia following total thyroidectomy. Otolaryngol Head Neck Surg 2012, 41:401-406.

19. Sahmkow SI, Audet N, Nadeau S, Camiré M, Beaudoin D: Outpatient thyroidectomy: safety and patients' satisfaction. Otolaryngol Head Neck Surg 2012,41:S1-12.

doi:10.1186/1916-0216-43-5

Cite this article as: Le et al:: Validation of 1-hour post-thyroidectomy parathyroid hormone level in predicting hypocalcemia. Journal of Otolaryngology - Head and Neck Surgery 2014 43:5.

\section{Submit your next manuscript to BioMed Central and take full advantage of:}

- Convenient online submission

- Thorough peer review

- No space constraints or color figure charges

- Immediate publication on acceptance

- Inclusion in PubMed, CAS, Scopus and Google Scholar

- Research which is freely available for redistribution 